\title{
World Health Organization 2018 Guidelines on Rabies Prophylaxis: Contentious Issues
}

\author{
MK Sudarshan
}

\begin{abstract}
The World Health Organization (WHO) recently reviewed the scientific advancements in the area of rabies prophylaxis and in 2018 issued new guidelines for consideration of the national governments for implementation. Following this, a Government of India (Gol) expert group met in January 2019 in Delhi and examined these WHO recommendations for implementation in India. In this article, the author has reviewed both the WHO and Gol recommendations and provides expert interpretation and opinion to both service providers in government institutions and private medical practitioners.

Keywords: Immunoglobulin, Literature review, Monoclonal antibody, Prophylaxis, Rabies, Vaccines.

Pediatric Infectious Disease (2019): 10.5005/jp-journals-10081-1226
\end{abstract}

\section{BACKGROUND}

Rabies is a viral zoonotic disease that is transmitted to man mostly following bite of a rabid animal. It results in fatal encephalomyelitis that is practically $100 \%$ fatal. Yet the disease is nearly $100 \%$ preventable with timely and correct use of currently available rabies vaccines and immunoglobulins or monoclonal antibodies. Consequently, if rabies exposure is life-threatening, rabies prophylaxis is life-saving. Worldwide, annually an estimated 59,000 persons are known to die of rabies of which 20,000 , i.e., about one third are from India alone. The disease results following bite, scratch, and lick by a rabid animal. The vast majority of rabies deaths in India are following dog bites (97\%), and only a small proportion are due to other animals viz. cats (2\%), mongoose, jackals, foxes, etc. The annual incidence of animal bites is about $2.5 \%$ in children, and about one third of rabies deaths are in children in the country.

The WHO, Geneva, Switzerland, following expert consultations and reviews periodically provides recommendations on rabies prophylaxis for member countries to adopt, and the recent guidelines were issued in 2018. In India, these recommendations were reviewed in January 2019 by a Gol national expert consultation held at National Centre of Disease Control, Delhi. The resultant revised/new national guidelines are to be issued soon by Gol.

\section{Review Results}

In this context, a critical appraisal and expert review of the WHO, 2018 guidelines on rabies prophylaxis and of the most recent Gol recommendations (of minutes of the meeting) are given below under the following headings.

1. Rabies postexposure prophylaxis (PEP)

2. Rabies pre-exposure prophylaxis (PrEP)

\section{Rabies PEP}

It involves three components, i.e., wound management, administration of rabies vaccine, and immunoglobulin (in severe/ category III exposures).

- Wound management: This involves immediate thorough flushing of bite wound/s with water and washing with soap for
Department of Community Medicine, Kempegowda Institute of Medical Sciences, Bengaluru, Karnataka, India

Corresponding Author: MK Sudarshan, Department of Community Medicine, Kempegowda Institute of Medical Sciences, Bengaluru, Karnataka, India, Phone: +91 9341231396, e-mail: mksudarshan@ gmail.com

How to cite this article: Sudarshan MK. World Health Organization 2018 Guidelines on Rabies Prophylaxis: Contentious Issues. Pediatr Inf Dis 2019;1(4):154-156.

Source of support: Nil

Conflict of interest: None

15 minutes followed by application of an antiseptic like betadine, dettol, savlon, and others.

- Postexposure prophylaxis regimens: WHO recommendations list of preferred PEP regimens and alternatives, all of which have been assessed for immunogenicity, clinical outcome, feasibility, and cost-effectiveness, is given in Table $1 .^{1}$

The national expert group observed lack of evidence with respect to this WHO recommendation in Indian settings and recommended multicentric studies to generate more evidence. ${ }^{2}$ Till such time, it is recommended to continue the current practice of updated Thai Red Cross (TRC) regimen (2-2-2-0-2 on days $0-3-7-28$ ) of 4 weeks duration in government institutions. Also, the current practice of five-dose Essen intramuscular (IM) regimen (1-1-1-1-1 on days $0-3-7-14-28$ ) will continue that is largely in vogue in private practice.

It must be noted here that even the labels/product inserts of the currently marketed rabies vaccines contain updated TRC intradermal (ID) regimen and five-dose Essen IM regimen. Hence, legally also it is wise to continue with these regimens. Consequently, the use of any other above WHO regimens will amount to "offlabel" use. The "off-label" use is to be strictly avoided in private practice, and in government institutions, it shall be done only after a necessary government/administrative order is issued for the purpose. 


\begin{tabular}{|c|c|c|}
\hline PEPregimen & $\begin{array}{l}\text { Duration of } \\
\text { course }\end{array}$ & $\begin{array}{l}\text { No. of injection sites } \\
\text { per clinic visit (days } 0 \text {, } \\
3,7,14 \text {, and } 21-28 \text { ) }\end{array}$ \\
\hline \multicolumn{3}{|c|}{ WHO-recommended ID regimen } \\
\hline 1 week, two sites & 7 days & $\begin{array}{l}2-2-2-0-0 \text { (days } \\
0-3-7)\end{array}$ \\
\hline \multicolumn{3}{|c|}{ WHO-recommended IM regimens } \\
\hline 2 weeks & $14-28$ days & $\begin{array}{l}1-1-1-1-0 \text { (days } \\
0-3-7-14-28)\end{array}$ \\
\hline 3 weeks & 21 days & $\begin{array}{l}2-0-1-0-1 \text { (days } \\
0-7-21 \text { ) }\end{array}$ \\
\hline \multicolumn{3}{|c|}{ Alternative immunogenic ID regimens } \\
\hline 1 month, two sites & $\leq 28$ days & $\begin{array}{l}2-2-2-0-2 \text { (days } \\
0-3-7-28)\end{array}$ \\
\hline 1 month, simplified four sites & $\leq 28$ days & $\begin{array}{l}4-0-2-0-1 \text { (days } \\
0-7-28)\end{array}$ \\
\hline 1 week, four sites & 7 days & $\begin{array}{l}4-4-4-0-0 \text { (days } \\
0-3-7)\end{array}$ \\
\hline
\end{tabular}

Postexposure Prophylaxis Following Re-exposure in Previously Vaccinated Individuals

Re-exposure less than 3 months of previous full-course PEP or PrEP (with documentary evidence): Only wound management and no PEP immunization (WHO) ${ }^{1}$

The Gol 2019 expert group endorsed this line of management ${ }^{2}$

Re-exposure more than 3 months of previous full-course PEP or PrEP: Wound management and PEP immunization (WHO $)^{1}$

The dosage is one vial by IM route or $0.1 \mathrm{~mL}$ by ID route Regimens $(\mathrm{WHO})^{1}$

1 site ID on days 0 and 3 (1-1-0-0-0);

or at 4 sites ID on day 0 (4-0-0-0-0);

or at 1 site IM on days 0 and 3 (1-1-0-0-0)

No rabies immunoglobulin (RIG) is needed, irrespective of category II or category III exposure.

However, the Gol 2019 expert group recommended continuation of the currently approved regimens of ID or IM vaccinations given on days 0 and $3 .^{2}$ Hence, it would be prudent to use the regimen approved by $\mathrm{Gol}$ and that on the label/product insert.

\section{Using Rabies Vaccines Labeled for IM Use by ID Route}

Rabies vaccines labeled for IM use can be used safely via the ID route, even if this constitutes "off-label" use (WHO).

The Gol expert group recommendation is awaited. Under these circumstances, it would be prudent not to go for any "offlabel" use of the rabies vaccines. This is controversial as in public health ID route only is used. Please clarify. [Of course, it is ID route in government institutions, but all rabies vaccines cannot be used by ID route unless the label permits its use by ID route, e.g., Speeda vaccine is by IM route only, and for its use by ID route, an official order is needed by the DHS for MO to give by ID route.] Note: Label refers to product insert/package insert/ vaccine literature, etc.

Evidence Suggests that a Change in the Route of Administration or in Vaccine Product during a PEP or PrEP Course is Safe and Immunogenic (WHO)

However, the Gol 2019 expert group approved the change in vaccine product, ${ }^{2}$ and approval for change in route of administration is awaited. The change in the vaccine product and route of vaccination may be done by the practitioner on a case-to-case basis to ensure completion of PEP that is life-saving.

\section{Prioritizing Rabies Exposures When Limited Amount of RIG is Available $(\mathrm{WHO})^{1}$}

If a limited amount of RIG is available, it should be prioritized for exposed patients on the basis of the following criteria:

- Multiple bites

- Deep wounds

- Bites to highly innervated parts of the body, such as the head, neck, and hands

- Severe immunodeficiency

- Bites from an animal with confirmed or probable rabies

- A bite, scratch, or exposure to mucous membranes from a bat (please add that bats in India are not infective for rabies)

Restricting RIG or vaccine to people with high-risk exposure to rabies may endanger those with lower risk exposure and should be considered carefully before being implemented.

In January 2019, the Gol expert group did not accept this recommendation and recommended continuation of the current practice that in all patients with category III exposure RIG should be given. ${ }^{2}$ Hence, no prioritization of cases for RIG use shall be practiced in India. Finally, in India, currently no PEP is recommended for exposure to bats.

\section{Using RIG for Only Wound Infiltration without Systemic Injection}

WHO no longer recommends injecting the remainder of the calculated RIG dose-IM at a distance from the wound. Instead, the calculated RIG dose can be fractionated in smaller, individual syringes to be used for several patients. This requires handling and storage in aseptic conditions. Unused fractionated doses and open vials of RIG should be discarded by the end of the day (WHO). ${ }^{3}$

However, in January 2019, the Gol expert group recommended continuation of the existing practice of injecting the remaining RIG volume intramuscularly, as close as possible to the presumed exposure site, to the degree that is anatomically feasible. ${ }^{2}$ It would be wise to go with the current practice of injecting the left over volume of RIG at a site away from the site of the vaccination, and this is mentioned even in the product insert/label.

\section{For Mucosal Exposure with No Wound, Rinsing with (Diluted ${ }^{3}$ ) RIG can be Considered (WHO)}

The Gol expert group recommendation is awaited. As rabies is practically $100 \%$ fatal, it would be prudent to use the WHOrecommended procedure in such cases that are rare in clinical practice.

Consumption of Meat or Milk from a Rabid Animal is Strongly Discouraged and should be Avoided but if it Occurs, PEP is Not Indicated (WHO). ${ }^{3}$ Milk that has been Pasteurized Presents No Risk for Rabies Virus Transmission (WHO) ${ }^{1}$

The Gol 2019 expert group endorsed this recommendation ${ }^{2}$ (for milk only and about meat it is silent). It would be OK to follow the WHO guidelines as this has been the topic of intense debate in many WHO meetings in the past. 
If Any Doses are Delayed, Vaccination should be Resumed and Not Restarted $(\mathrm{WHO})^{3}$

Even the Gol 2019 expert group has endorsed this recommendation. ${ }^{2}$ This shall be followed in clinical practice. A few days of delay does not matter, and it is very important to complete the initial threedose series of vaccination, i.e., given on days 0,3 , and 7 within 7 days or as close to that as possible as it is the primary and priming course of PEP vaccination. The subsequent two doses given on days 14 and 28 by IM route (or one dose given on day 28 by ID route) are immunologically the booster dose/s and ensure completion of the PEP regimen. [This is in contradiction to Indian Academy of Pediatrics (IAP) recommendations.]

People Who have Received at Least Two Doses (ID or IM) of a Cell Culture Vaccine on an Appropriate Schedule before Discontinuation should be Considered as Having Received $\operatorname{PrEP}(W H O)^{3}$

The Gol expert group recommendation is awaited. This may be considered by the physician on an individual case-to-case basis based on the assessment of rabies risk in the given bitten/rabies exposed individual.

\section{If Available, the Use of mAb Products Instead of RIG is} Encouraged $(\mathrm{WHO})^{3}$

In October 2017, Serum Institute of India Pvt. Ltd., Pune, launched the world's first rabies monoclonal antibody (rmAb) and is now available in the market.

The Gol expert group recommended that rmAb needs to be studied for its effectiveness, safety in multicentric Indian settings. ${ }^{2}$ However, as this indigenous product is legally and commercially available, it may be freely used in private practice and when made available in government institutions.

\section{Pre-exposure Prophylaxis Regimens}

The WHO-recommended PrEP regimens are given in Table 2., ${ }^{1,3}$

In January 2019, the Gol expert group, however, recommended continuation of the currently approved three-dose regimen ( $0.1 \mathrm{~mL}$ ID at one site or 1 vial IM given on days 0,7 , and 21 or 28$)^{2}{ }^{2}$ The medical practitioner may consider using any of the above WHO regimens on a case-to-case basis.

\section{What is "off-label" Use?}

All medicines, including rabies vaccines and immunoglobulins/ rmAb are accompanied by product inserts (or also known as labels or enclosures, etc.) that provide guidance for their use in the
Table 2: WHO-recommended PrEP regimens

\begin{tabular}{|c|c|c|}
\hline PrEP regimen & Duration of course & $\begin{array}{l}\text { No. of injection sites } \\
\text { per clinic visit (days } 0 \text {, } \\
3,7,14,21-28 \text { ) }\end{array}$ \\
\hline \multicolumn{3}{|l|}{$\begin{array}{l}\text { WHO-recommended } \\
\text { ID regimen }\end{array}$} \\
\hline Two visits two sites & 7 days & 2-0-2-0-0 (days 0-7) \\
\hline \multicolumn{3}{|l|}{$\begin{array}{l}\text { WHO-recommended } \\
\text { IM regimen }\end{array}$} \\
\hline Two visits one site & 7 days & $1-0-1-0-0$ (days 0-7) \\
\hline \multicolumn{3}{|c|}{$\begin{array}{l}\text { PrEP under specific circumstances This should be deleted as it } \\
\text { says 1-day course is not considered complete unless second dose } \\
\text { is given within } 1 \text { year of first dose and in case of exposure before } \\
\text { second dose full PEP has to be practiced. It is the recommendation } \\
\text { of WHO. If you read below, the Gol has not endorsed it }\end{array}$} \\
\hline Single visit, ID & 1 day & $2-0-0-0-0$ \\
\hline Single visit, IM & 1 day & $1-0-0-0-0$ \\
\hline
\end{tabular}

patients. These labels or product inserts are approved by the Drug Controller General of India. Any usage that involves deviation from the guidelines provided in these product inserts/labels is considered as "off-label" use. The private medical practitioners in particular must avoid "off-label" use as in the event of any mishap, as they are liable to be sued under consumer protection act.

\section{Conclusion}

In government institutions and in private practice, the recommendations of $\mathrm{Gol}$ and the guidelines given in the product (rabies vaccines, RIG and rmAb) insert shall be followed. Any use of WHO recommendation that is not endorsed by Gol or in the product insert may be made only in exceptional circumstances and solely with the intention of saving the life of the patient and it shall be defendable in a court of law, if the need arises. Finally, the Gol national guidelines on rabies prophylaxis are expected to be issued soon this year.

\section{References}

1. WHO, WHO expert consultation on rabies, Technical Report Series, 1012, 2018, Geneva, Switzerland.

2. Government of India. National Centre for Disease Control, expert group meeting to review the national guidelines on rabies prophylaxis, 8th January, 2019, Minutes of the meeting, New Delhi.

3. WHO, Rabies vaccines: WHO position paper - April, 2018, Weekly epidemiological record, no.16, 93, Geneva, Switzerland. 